\title{
Release properties of atelocollagen-gelatin complexes as carriers for local administration of fluvastatin
}

\author{
Koichi KIDA ${ }^{1,2}$, Koji TANABE²,3, Hodaka SASAKI ${ }^{1,2}$, Yoshitaka FURUYA ${ }^{1,2}$, Tadashi MIURA², Masao YOSHINARI ${ }^{2}$ \\ and Yasutomo YAJIMA ${ }^{1,2}$ \\ ${ }^{1}$ Department of Oral and Maxillofacial Implantology, Tokyo Dental College, 2-9-18, Misaki-cho, Chiyoda-ku, Tokyo 101-0061, Japan \\ ${ }^{2}$ Division of Oral Implants Research, Oral Health Science Center, Tokyo Dental College, 2-9-18, Misaki-cho, Chiyoda-ku, Tokyo 101-0061, Japan \\ ${ }^{3}$ Department of Pharmacology, Tokyo Dental College, 2-9-18, Misaki-cho, Chiyoda-ku, Tokyo 101-0061, Japan \\ Corresponding author, Tadashi MIURA; E-mail: tamiura@tdc.ac.jp
}

\begin{abstract}
The aim of this study was to investigate properties of atelocollagen/gelatin complexes (AC/Gel) and their characteristics of sustained statin release, to assess the utility of $\mathrm{AC} / \mathrm{Gel}$. AC/Gel were prepared by changing the mixing ratio of $\mathrm{AC}$ (0 to $40 \%$ of $\mathrm{AC})$. Analysis of spectra of fluvastatin (Flu), gelatin (Gel), and Flu with Gel complex using a Fourier transform-infrared spectrometer indicates that Flu was bound to Gel through a bond involving the carboxyl and amino groups. Evaluation of characteristics of sustained release of Flu from the AC/Gel using an ultraviolet-visible spectrophotometer showed that the release rate of Flu decreased with increasing the AC content. The histological evaluation using of Sprague-Dawley rats suggest that, unlike the pure Gel sponge, the AC/Gel was not absorbed in an early stage. Therefore, the present study showed that sustained Flu release can be controlled by using an AC/Gel, suggesting the utility of this composite material.
\end{abstract}

Keywords: Fluvastatin, Atelocollagen, Gelatin, Controlled release, Regeneration

\section{INTRODUCTION}

Statins, which are competitive inhibitors of the 3-hydroxy-3-methylglutaryl coenzyme A (HMG$\mathrm{CoA})$ reductase enzyme, are widely used to suppress cholesterol synthesis for the treatment of hyperlipidemia. In addition to the cholesterol-lowering effect, statins increase BMP-2 expression, decreasing the activity of osteoclasts and stimulating osteoblast differentiation, promoting bone formation ${ }^{11}$. Recently, the promoting effect of statins on bone formation has drawn attention to the field of dentistry and has been extensively studied for possible applications, such as accelerating healing at extraction sockets and implant sites $^{2,3)}$.

Systemic administration of high-dose statins may increase the risk of side effects, such as rhabdomyolysis and myopathy ${ }^{4}$. Therefore, local administration of statins is being attempted to avoid the risk of side-effects. However, simple direct administration of statins to the affected sites is not effective for osteoinduction because the drug diffuses into the surrounding tissues ${ }^{5-7)}$.

Accordingly, controlled release systems for statins are required for local administration of statins with carriers, such as gelatin, atelocollagen sponge (Teruplug $\left.{ }^{\circledR}\right), \alpha$-TCP, $\beta$-TCP, HA, and PLGA ${ }^{8-11)}$. In particular, gelatin is widely used as a drug carrier because of desirable characteristics including (1) proven safety, (2) wide application of this biodegradable material for clinical purposes, (3) different physical properties by chemical modification, and (4) ease of

Color figures can be viewed in the online issue, which is available at J-STAGE.

Received May 11, 2016: Accepted Nov 21, 2016

doi:10.4012/dmj.2016-179 JOI JST.JSTAGE/dmj/2016-179 attaching to drugs ${ }^{12-15)}$. Moreover, gelatin derivatives obtained by acid or alkali treatment have different isoelectric points. Such gelatin derivatives are capable of carrying positively or negatively charged substances via electrostatic interactions ${ }^{16)}$.

In our earlier studies, we have investigated fluvastatin sodium, an amphiphilic statin that has both membrane permeability and water solubility ${ }^{17}$, which have the properties that are advantageous for local administration. We conjugated fluvastatin sodium to a nontoxic, thermally crosslinked gelatin sponge and showed that the resulting sponge-form conjugates promoted bone formation in vivo ${ }^{18-20}$. However, gelatin is water-soluble, and the thermal crosslinking treatment could prolong dissolution time by only approximately 3 days ${ }^{20)}$. Therefore, the development of carriers capable of more stable drug release is required.

In the present study, we focused on atelocollagen as a statin carrier. Collagen, which forms gelatin on partial hydrolysis, has lower susceptibility to proteases than gelatin ${ }^{21)}$ because of its distinctive triple helix structure. Atelocollagen is a collagen derivative from which collagen components responsible for antigenicity have been removed, providing it a greater potential as a biomaterial ${ }^{22}$. Since collagen is easy to aggregate in an aqueous phase because of its conformation, it is more hydrophobic than water-soluble gelatin. Therefore, it appeared impossible to immobilize fluvastatin on atelocollagen by electrostatic interactions. Accordingly, we decided to prepare an atelocollagen/gelatin complex in which gelatin functioned as the fluvastatin carrier, as in our earlier studies, and to make efficient use of atelocollagen for retarding degradation of the carrier. 
Prior to the clinical application of the atelocollagen/ gelatin complex as a statin carrier, it is necessary to determine (1) properties of the atelocollagen/gelatin complex (internal structure, statin-binding mechanism, and bio-absorbability) and (2) characteristics of sustained release of the statin from the atelocollagen/ gelatin complex. However, no relevant previous reports were available.

Therefore, the present study aimed to investigate properties of atelocollagen/gelatin preparations and their characteristics of sustained statin release, to assess the utility of this class of complex.

\section{MATERIALS AND METHODS}

\section{Atelocollagen/gelatin complexes preparation}

Fluvastatin sodium salt (Flu; Lot WEG3258, Toronto Research Chemicals, Ontario, Canada), alkaline gelatin derived from porcine (Gel; isoelectric point of 7.0-9.0, Nitta Gelatin, Osaka, Japan) and type 1 atelocollagen derived from bovine (AC; Lot No.818025, Koken, Tokyo, Japan) were used in this study.

Three kinds of solutions were prepared as shown below; Solution A: $90 \mathrm{mg}$ of $\mathrm{AC}$ was dissolved to 10 $\mathrm{mL}$ of final $100 \mathrm{mM}$ phosphoric acid (0.9 AC solution). Solution B: Mixture of $90 \mathrm{mg}$ of Gel and $870 \mu \mathrm{M}$ Flu was dissolved to $10 \mathrm{~mL}$ of the phosphoric acid $(0.9 \%$ Gel solution with Flu) according to the early study ${ }^{18)}$. Solution C: $90 \mathrm{mg}$ of Gel was dissolved to $10 \mathrm{~mL}$ of the phosphoric acid (0.9\% Gel solution without Flu). The above solutions of $\mathrm{A}$ and $\mathrm{B}$ as Flu containing group, or of $\mathrm{A}$ and $\mathrm{C}$ without Flu group, were mixed in a mixing ratio of the solution A, as describing in Table 1. Each solution was mixed thoroughly, continuous stirring for $48 \mathrm{~h}$ at $4^{\circ} \mathrm{C}$. For preparing group with Flu, solutions of A and B were mixed, named F-AC0, 10, 20, 30, and 40 (The concentration of Flu involved in F-AC0, 10, 20, 30, and 40 were $870,783,696,609,522 \mu \mathrm{M}$, respectively). For preparing group without Flu, solution of $\mathrm{A}$ and $\mathrm{C}$ were mixed named AC0, 10, 20, 30, and 40. The mixture was injected in a cylindrical container having an inner diameter of $16 \mathrm{~mm}$ and a height of $17 \mathrm{~mm}$, and then was frozen at $-20^{\circ} \mathrm{C}$ for $2 \mathrm{~h}$. Frozen samples were placed in a freeze dryer $\left(\right.$ FREEZONE $^{\circledR} 2.5$, LABCONCO, Kanzas city, MO, USA) and dried under vacuum at $-50^{\circ} \mathrm{C}$ for 24 $\mathrm{h}$ to prepare dry sponges. Finally, atelocollagen/gelatin complex were polymerized by cross-linking the sponge with heat treatment at $125^{\circ} \mathrm{C}$ for $30 \mathrm{~min}$.

Observation with a scanning electron microscope (SEM) Fabricated complexes were observed using a scanning electron microscope (SU-6600, Hitachi, Tokyo, Japan). Each complex used for SEM specimen (F-AC0, 10, 20, 30 , and 40) was cut in thin sections with a razor blade. Before SEM observation, the cross-sectioned complexes were coated with Au-Pd using a sputter coater (SC500A, Elminet, Tokyo, Japan).

Fourier transform-infrared spectroscopy (FT-IR)

Spectra of Flu, Gel, and F-AC0 were obtained by a Fourier transform-infrared spectrometer (FT-IR430, JASCO, Tokyo, Japan). FT-IR spectra were obtained from discs containing $1 \mathrm{mg}$ sample in approximately $100 \mathrm{mg}$ potassium bromide $(\mathrm{KBr})$. The measuring was performed at a resolution of $1 \mathrm{~cm}^{-1}$ for 100 times iterations in the wavelength range from 4,000 to $400 \mathrm{~cm}^{-1}$. The obtained spectra $\left(1,600-1,500 \mathrm{~cm}^{-1}\right)$ were analyzed using a software (KnowItAll Informatics System, Bio-Rad, Hercules, CA, USA).

\section{Release test of fluvastatin from the complexes}

The complexes containing Flu (F-AC0 to 40) was used as experimental groups and complexes without Flu (AC0 to 40) were used as control groups (each complex $n=6$ ). Each complex was filled into $2 \mathrm{~mL}$ of phosphate buffered saline (PBS), and then they were stored in a dark box at $37^{\circ} \mathrm{C}$. After sampling $60 \mu \mathrm{L}$ of each solution eluted from the complex, released Flu from the complexes were measured with absorbance of $303 \mathrm{~nm}$ using an ultraviolet-visible spectrophotometer (V-660, JASCO) at $6 \mathrm{~h}, 24 \mathrm{~h}$, and then every day until 1 week from starting of release test. The dissolution rate was defined as absorbance ratio of eluate in each complex at appropriate period against initial F-AC solution before forming the complex. Ratio was calculated according to the following formula. Dissolution rate $(\%)=($ Absorbance

Table 1 Components of atelocollagen/gelatin complex

\begin{tabular}{|c|c|c|c|c|c|}
\hline $\begin{array}{c}\text { AC/Gel complex } \\
\text { with Flu }\end{array}$ & $\begin{array}{c}\mathrm{A} \\
0.9 \% \mathrm{AC} \text { solution } \\
(\%)\end{array}$ & $\begin{array}{c}\text { B } \\
\text { 0.9\% Gel solution } \\
\text { with Flu } \\
(\%)\end{array}$ & $\begin{array}{l}\text { AC/Gel complex } \\
\text { without Flu }\end{array}$ & $\begin{array}{c}\mathrm{A} \\
0.9 \% \mathrm{AC} \text { solution } \\
(\%)\end{array}$ & $\begin{array}{c}\mathrm{C} \\
0.9 \% \text { Gel solution } \\
\text { without Flu } \\
(\%)\end{array}$ \\
\hline F-AC40 & 40 & 60 & AC40 & 40 & 60 \\
\hline F-AC30 & 30 & 70 & AC30 & 30 & 70 \\
\hline F-AC20 & 20 & 80 & $\mathrm{AC} 20$ & 20 & 80 \\
\hline F-AC10 & 10 & 90 & $\mathrm{AC} 10$ & 10 & 90 \\
\hline F-AC0 & 0 & 100 & $\mathrm{AC} 0$ & 0 & 100 \\
\hline
\end{tabular}


of eluate collected in each section/Absorbance of initial F-AC solution of each complex) $\times 100$.

\section{Bioabsorbable experiment}

Fifteen-week-old, male, Sprague-Dawley rats $(n=3)$ obtained from Sankyo Laboratory (Tokyo, Japan) were used in the experiment. The rats were allowed food and water ad libitum and maintained on a $12 \mathrm{~h}$ light/ dark cycle (lights on from 8:00 to $20: 00$ ) at $23 \pm 1^{\circ} \mathrm{C}$ with $60 \pm 10 \%$ humidity during the experiment.

All rats, after isoflurane (Wako, Osaka, Japan) inhalation pentobarbital sodium (Somnopentyl, Kyoritsu Seiyaku, Tokyo, Japan) were anesthetized by intraperitoneal injection of $0.7 \mu \mathrm{L} / \mathrm{g}$ body weight. Thigh was blunt dissection of muscle added after incision that has been shaved. After removing the periosteum to expose the femur, a $1.6 \mathrm{~mm}$ round bur was used to create a cylindrical bone defect with $1.6 \mathrm{~mm}$ in diameter and depth under constant irrigation with normal saline solution to prevent overheating of the bone edges on either side femoral central portion. The complexes were cut into pieces $1.6 \mathrm{~mm}$ in diameter and height to fit the bone defect area.

After hemostasis, AC40 complex without Flu as an experimental group, or AC0 as a control group, was implanted into the defect site, and the site was sutured. All animal experiments in this study were conducted in accordance with the Tokyo Dental College Guidelines for Animal Experimentation (Approval date: 4/1/2015; Approval number: 273002).

The rats were humanely sacrificed with intraperitoneally administered overdoses of pentobarbital at 3,5 , and 7 days after surgery. The removed femurs were immersed in $10 \%$ neutral buffered formalin for 7 days, and paraffin specimens were made after decalcification with 10\% EDTA $(\mathrm{pH}$ 7.0-7.5) (Wako) for 7 days at room temperature. The specimens were sliced into $3 \mu \mathrm{m}$-thick sections and stained with hematoxylin and eosin staining (H-E staining) according to standard protocols. These samples were morphologically observed using a universal photomicroscope (Axiophot 2, Carl Zeiss, Oberkochen, Germany).

\section{Statistical analysis}

All data are expressed as the mean \pm standard deviation (SD). The data were analyzed using a statistical analysis software package (GraphPad Prism6, MDF, Tokyo, Japan). Data were first analyzed by one-way ANOVA. When this analysis suggested a significant difference between groups $(p<0.01)$, the data were further analyzed by the Tukey post hoc multiple comparison tests.

\section{RESULTS}

Observation with a scanning electron microscope (SEM) SEM images of complexes of F-AC0, 10, 20, 30, and 40, shown in Fig. 1 revealed that all preparations had an internal network structure composed of continuous microscopic pores of approximately $200 \mu \mathrm{m}$.

\section{Fourier transform-infrared spectroscopy (FT-IR)}

The results of FT-IR analysis is shown in Fig. 2. Characteristic peaks of Gel and Flu were confirmed, and a mixture of the two, F-AC0, showed changes in peaks. In Fig. 2, the spectra are shown focusing on the $1,600-1,500 \mathrm{~cm}^{-1}$ range, in which marked peak changes are observed. The peak at $1,587 \mathrm{~cm}^{-1}$, a carboxyl group-
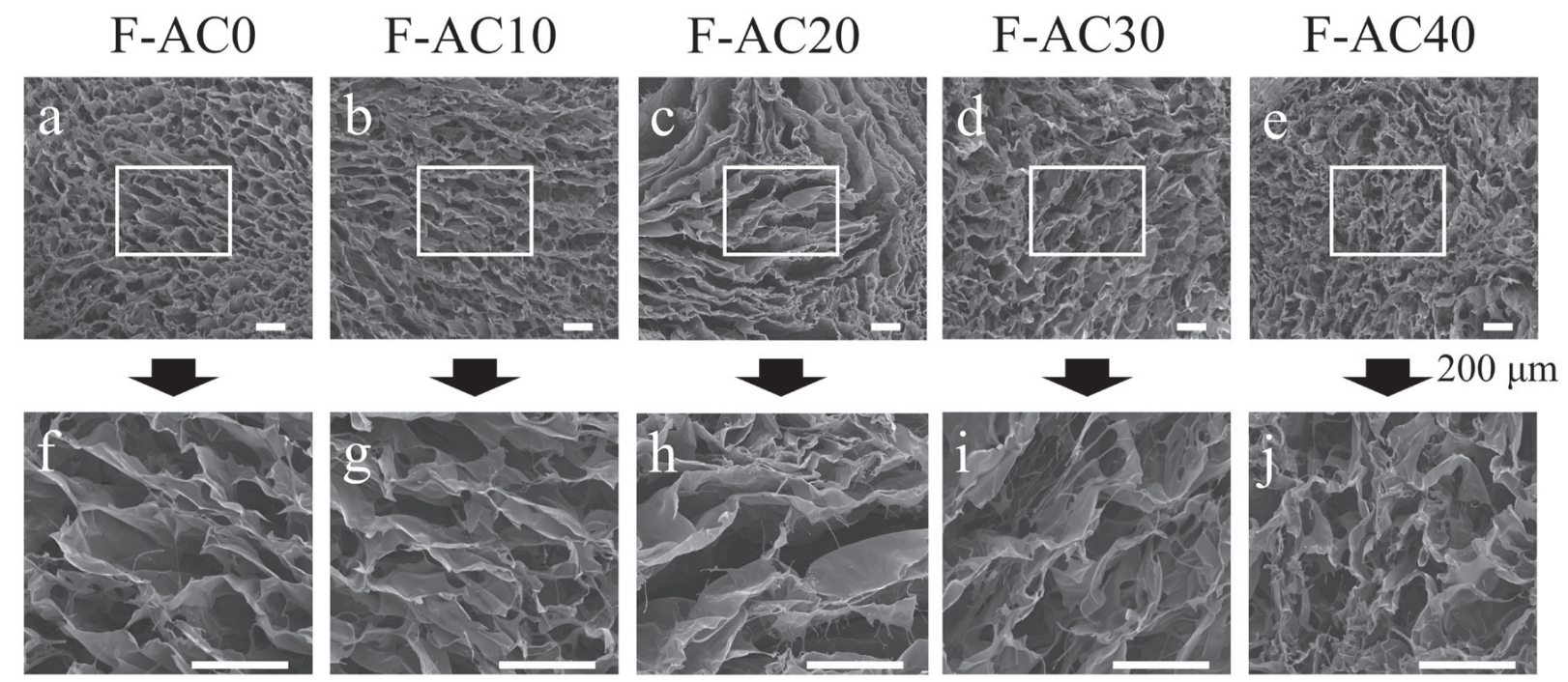

$200 \mu \mathrm{m}$

Fig. 1 SEM images of each complex of F-AC0, 10, 20, 30, and 40.

(a)-(e) were setting at magnification $\times 60$. (f)-(j) were setting at magnification $\times 200$. Scale bars show length of $200 \mu \mathrm{m}$. 


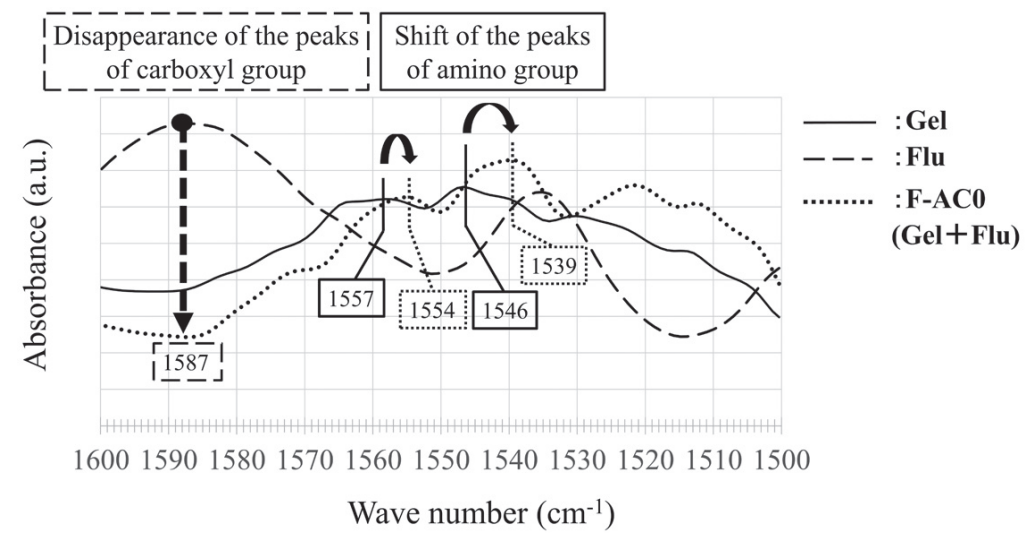

Fig. 2 FT-IR analyses ranging from 1,600 1,500 $\mathrm{cm}^{-1}$.

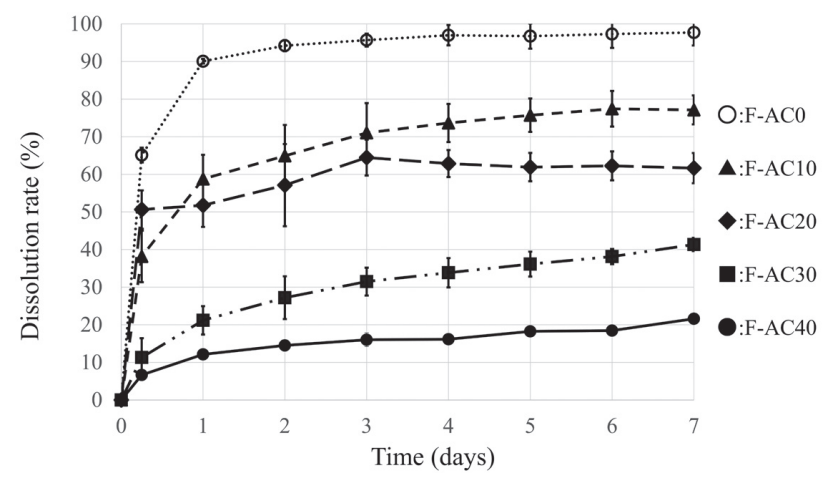

Fig. 3 Dissolution rate of the each complex at 6 h, 24 h, and then every day until 1 week from starting of release test.

Error bars represent means \pm standard deviation for $n=6$.

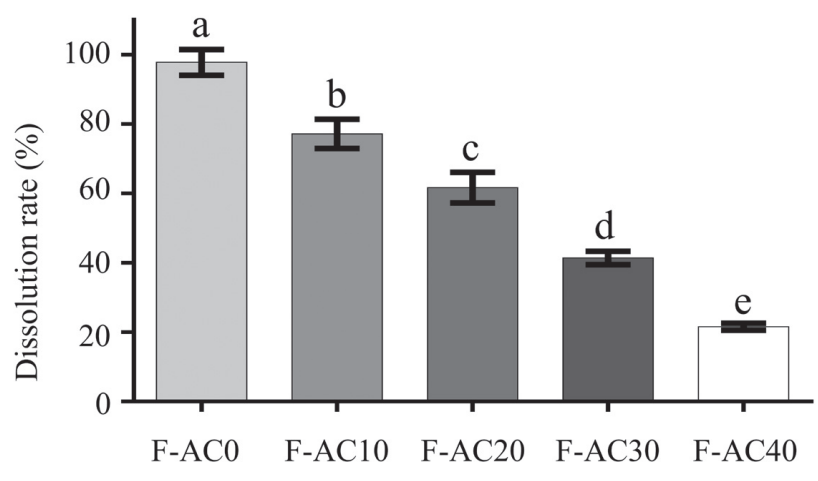

Fig. 4 Dissolution rate of each complex containing Flu. One-way ANOVA Tukey's multiple comparisons tests $(n=6)$. Significant differences were observed between each complexes $(p<0.01)$. Different alphabetic character (a-e) indicates significant differences.

with increasing $\mathrm{AC}$ content. On the control $\mathrm{AC} 0,10$, 20, 30, and 40 groups not containing Flu, no noticeable absorbance at wavelength of $303 \mathrm{~nm}$ was observed. With regard to F-AC0, the percentage of elution approached $90 \%$ on the first day and gradually increased. With regard to F-AC10 and 20 with low AC content, the dissolution rate were 59 and $52 \%$, respectively, on the first day and gradually increased to 77 and $62 \%$ thereafter. With regard to F-AC30 and 40 with high AC content, the dissolution rate gradually increased from 21 and $12 \%$ elution at day 1 to 41 and $22 \%$ at day 7 , respectively.

\section{Bioabsorbable experiment}

The complex absorption process in animal experiments is shown in Fig. 5. No abnormal bleeding or inflammation was observed in any animal experiment that was conducted. In animals that received AC0, Gel sponge residues were observed on postoperative day 3. No sponge structure was visible on postoperative $(p<0.01)$, and the release rate significantly decreased 

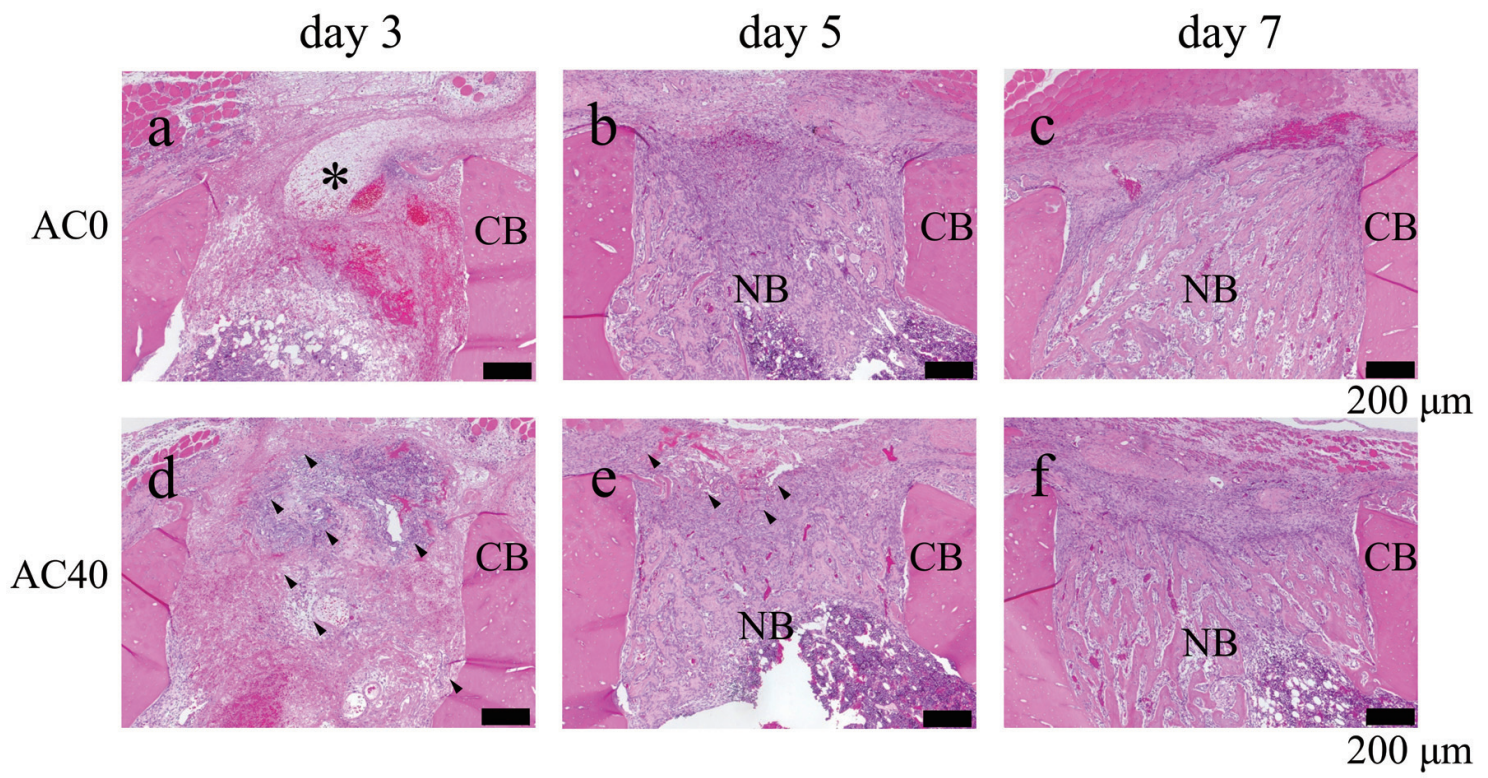

\section{*: gelatin $\quad$ : atelocollagen CB: cortical bone NB : new bone}

Fig. 5 Histological analysis of absorption of complexes of AC0 (a-c) and those of AC40 (d-f) in femoral at day $3(\mathrm{a}, \mathrm{d}), 5$ (b, e), and 7 (c, f).

In animals that received AC0, Gel sponge residues were observed on postoperative day 3 (a). No sponge structure was visible on postoperative day 5 or 7 (b, c). In animals that received AC40, residual AC fibers intensively stained with hematoxylin were observed on postoperative day 3 (d). The residual fibers were yet visible on postoperative day 5, and they were completely absorbed on day 7 (e, f). Scale bars: $200 \mu \mathrm{m}$.

day 5 or 7. In animals that received AC40, residual AC fibers intensively stained with hematoxylin were observed on postoperative day 3. The residual fibers were yet visible on postoperative day 5 , and they were completely absorbed on day 7 .

\section{DISCUSSION}

In the present study, we prepared and characterized atelocollagen/gelatin complexes as carriers for controlled, sustained statin release.

In our earlier studies, we used a Gel sponge carrying Flu via electrostatic interactions ${ }^{18-20}$. The dissolution rate of this Gel sponge is controllable for up to 3 days by thermal crosslinking ${ }^{20)}$. However, no conventional thermal crosslinking methods were effective in further prolonging the dissolution time of the Gel sponge. AC used in this study, from which antigenic telopeptides have been removed by proteolytic treatment, is highly biocompatible and has been widely used in research and clinical settings ${ }^{22)}$. Although collagen and gelatin are identical to each other in primary structure (amino acid composition), they differ in secondary structure, and collagen, which forms gelatin on partial hydrolysis, has lower susceptibility to proteases than gelatin ${ }^{21)}$ because of its three-dimensional structure. Therefore, atelocollagen was adopted as a biodegradable carrier with a reduced rate of dissolution, even without undergoing harsh crosslinking treatment, which is required for gelatin use. However, the fibrillar protein collagen has a hydrophobic character and was considered to be incompatible with the electrostatic interaction-based method used to attach Flu in our earlier study. Although other studies have used simvastatin-impregnated atelocollagen sponge $\left(\text { Teruplug }^{\circledR}\right)^{9,14,23)}$, these reports do not describe statin binding by collagen or characterize the time course of statin release. In the present study, we accordingly prepared an atelocollagen/gelatin complex in which Gel was used as a carrier of Flu, as in our earlier studies, and $\mathrm{AC}$ was used to retard the degradation of the carrier.

When gelatin is used as a carrier, the solubility is controlled by the rate of crosslinking. Crosslinking is accomplished using chemicals such as formaldehyde ${ }^{24)}$ and glutaraldehyde ${ }^{25)}$ or physical methods such as UV irradiation $^{26)}$ and heating ${ }^{18-20)}$. However, it has been reported that with chemical crosslinking, cytotoxicity of residual reactants from the chemical treatment causes issues ${ }^{24,25,27)}$. Because nontoxic, thermally-crosslinked gelatin was successfully used in our earlier studies, we used thermal crosslinking in the present study.

SEM observations showed no marked structural differences among complexes prepared from F-AC0 (a conventional Gel sponge) and F-AC10, 20, 30, and 40, with a porous structure consisting of approximately $200 \mu \mathrm{m}$ pores observed for all analyzed materials. The 
pore size and porosity of sponge-like scaffolds used in regenerative therapy markedly affect cell proliferation, and approximate pore diameters ranging from 200 to $350 \mu \mathrm{m}$ have been reported to be effective for bone tissue regeneration $^{28-30)}$. Therefore, the atelocollagen/gelatin complexes prepared in this study may be applicable as scaffolds for bone tissue regeneration.

The infrared spectroscopic analysis in this study showed that, in F-AC0 spectra, the peak at 1,587 $\mathrm{cm}^{-1}$ derived from the $\mathrm{Flu}^{31)}$ carboxyl group was absent and the peaks at 1,557 and $1,546 \mathrm{~cm}^{-1}$ from the amino group of $\mathrm{Gel}^{32)}$ shifted to 1,554 and $1,539 \mathrm{~cm}^{-1}$. This result indicates that Flu was bound to Gel through a bond involving the carboxyl and amino groups. These results showed that Flu and Gel were bound via electrostatic interactions, as we expected, because Flu is negatively charged at approximately neutral $\mathrm{pH}$ and that positively charged basic Gel was used in this study.

The present characterization of Flu release from atelocollagen/gelatin complexes showed that the sustained Flu release rate decreased with increasing the $\mathrm{AC}$ content. Concentration of Flu in this experiment is different for each complex. AC is soluble only in an acidic solution. On the other hand, Flu is precipitated in the acidic conditions. Therefore, it was impossible to add Flu in the dissolved state in AC solution. Maximum amount of Flu, which was capable to bind with each Gel solution, was mixed to Gel for preparation of F-AC. In our preliminary experiment, Flu was confirmed not to bind to AC. The complex prepared from Gel alone (F-AC0) was found to release $90 \%$ of Flu in 1 day. Previous reports have documented that the release of a physiologically active substance from a carrier occurs in two stages (i.e., carrier diffusion and erosion), which can occur in steps or simultaneously ${ }^{33,34)}$. Similarly, it is likely that statin diffusion from F-AC0 and disintegration of the sponge itself concurrently occurred in our experiment, resulting in the rapid elevation of the release rate. In contrast, complexes containing a low proportion of $\mathrm{AC}$ (F-AC10 and 20) released $\geq 50 \%$ Flu in 1 day with a subsequent gradual increase of the release rate. Complexes with high AC content (F-AC30 and 40) gradually released Flu over 7 days without rapid release in an early phase that was observed for other materials. Based on previous reports, these observations may be explained as follows. The initial release of a physiologically active substance caused by diffusion of a carrier, time required for transition to the decompositiondriven second stage of the release, and the release rate in that stage depend on the crosslink density of the carrier $^{33,34)}$. Other researchers have shown that the strength of collagen can be enhanced by crosslinking ${ }^{35)}$, similar to that of gelatin. Therefore, it is likely that the release properties of Flu were prolonged with increasing the $\mathrm{AC}$ contents and the degree of crosslinking.

The present in vivo study showed that the sponge prepared of Gel alone (AC0) remained visible for 3 days at the implant site and disappeared 5 days after implantation. However, in animals that received AC40, AC remained visible for 5 days after surgery and disappeared completely on day 7 . These results suggest that, unlike the pure Gel sponge, the atelocollagen/gelatin complex was not absorbed in vivo in an early stage following the implantation and may support sustained drug release for an extended period. Controlled release of Flu in vivo is believed to take place appropriately after inflammatory phase. Further investigations are necessary to examine optimal concentration of Flu and $\mathrm{AC} / \mathrm{Gel}$ ratio in vivo.

In summary, this study showed that atelocollagen/ gelatin complex had porosity similar to conventional carriers and confirmed bonding between Flu and Gel. The Flu release test showed that the release rate decreased as the AC content increased. These results suggest that novel atelocollagen/gelatin complex may be clinically useful carriers to accomplish local sustained Flu release for acceleration of bone formation. Therefore, the present study showed that sustained Flu release can be controlled using an atelocollagen/gelatin complex, suggesting the utility of this composite material.

\section{ACKNOWLEDGMENT}

This work was supported by JSPS KAKENHI Grant Number JP15K20488.

\section{REFERENCES}

1) Mundy G, Garrett R, Harris S, Chan J, Chen D, Rossini G, Boyce B, Zhao M, Gutierrez G. Stimulation of bone formation in vitro and in rodents by statins. Sci Am 1999; 286: 19461949.

2) Ayukawa Y, Okamura A, Koyano K. Simvastatin promotes osteogenesis around titanium implants: A histological and histometrical study in rats. Clin Oral Implants Res 2004; 15 : 346-350.

3) Wu Z, Liu C, Zang G, Sun H. The effect of simvastatin on remodelling of the alveolar bone following tooth extraction. Int J Oral Maxillofac Surg 2008; 37: 170-176.

4) Ogasawara R, Furuya Y, Sasaki H, Yoshinari M, Yajima Y. Original effects of oral administration of simvastatin on bone formation in senile osteoporosis rat. J Hard Tissue Biol 2013; 22: 461-472.

5) Fujimura K, Bessho K, Kusumoto K, Ogawa Y, Iizuka T. Experimental studies on bone inducing activity of composites of atelopeptide type I collagen as a carrier for ectopic osteoinduction by rhBMP-2. Biochem Biophys Res Commun 1995; 208: 316-322.

6) Bessho K, Iizuka T. Activity and solubility of bone morphogenetic protein derived from porcine bone matrix. $\mathrm{Br}$ J Oral Maxillofac Surg 1994; 32: 86-90.

7) Kempen DHR, Lu L, Hefferan TE, Creemers LB, Maran A, Classic KL, Dhert WJA, Yaszemski MJ. Retention of in vitro and in vivo BMP-2 bioactivities in sustained delivery vehicles for bone tissue engineering. Biomaterials 2008; 29: 32453252.

8) Fukui T, Ii M, Shoji T, Matsumoto T, Mifune Y, Kawakami Y, Akimaru H, Kawamoto A, Kuroda T, Saito T, Tabata Y, Kuroda R, Kurosaka M, Asahara T. Therapeutic effect of local administration of low-dose simvastatin-conjugated gelatin hydrogel for fracture healing. J Bone Miner Res 2012; 27: 1118-1131.

9) Alam S, Ueki K, Nakagawa K, Marukawa K, Hashiba Y, Yamamoto E, Sakulsak N, Iseki S. Statin-induced bone morphogenetic protein (BMP) 2 expression during bone 
regeneration: an immunohistochemical study. Oral Surg Oral Med Oral Pathol Oral Radiol Endod 2009; 107: 22-29.

10) Rojbani H, Nyan M, Ohya K, Kasugai S. Evaluation of the osteoconductivity of $\alpha$-tricalcium phosphate, $\beta$-tricalcium phosphate, and hydroxyapatite combined with or without simvastatin in rat calvarial defect. J Biomed Mater Res A 2011; 98A: 488-498.

11) Masuzaki T, Ayukawa Y, Moriyama Y, Jinno Y, Atsuta I, Ogino Y, Koyano K. The effect of a single remote injection of statin-impregnated poly(lactic-co-glycolic acid) microspheres on osteogenesis around titanium implants in rat tibia. Biomaterials 2010; 31: 3327-3334.

12) Einerson N, Stevens KR, Kao WJ. Synthesis and physicochemical analysis of gelatin-based hydrogels for drug carrier matrices. Biomaterials 2003; 24: 509-523.

13) Hokugo A, Ozeki M, Kawakami O, Sugimoto K, Mushimoto K, Morita S, Tabata Y. Augmented bone regeneration activity of platelet-rich plasma by biodegradable gelatin hydrogel. Tissue Eng 2005; 11: 1224-1233.

14) Mukozawa A, Ueki K, Marukawa K, Okabe K, Moroi A, Nakagawa K. Bone healing of critical-sized nasal defects in rabbits by statins in two different carriers. Clin Oral Implants Res 2011; 22: 1327-1335.

15) Shah SR, Werlang CA, Kasper FK, Mikos AG. Novel applications of statins for bone regeneration. Natl Sci Rev 2015; 2: 85-99.

16) Tabata Y, Hijikata S, Ikada Y. Enhanced vascularization and tissue granulation by basic fibroblast growth factor impregnated in gelatin hydrogels. J Control Release 1994; 31: 189-199.

17) Hamelin BA, Tutreon J. Hydrophilicity/lipophilicity: relevance for the pharmacology and clinical effects of HMGCoA reductase inhibitors. Trends Pharmacol Sci 1998; 19: 2637.

18) Tanabe K, Nomoto H, Okumori N, Miura T, Yoshinari M. Osteogenic effect of fluvastatin combined with biodegradable gelatin-hydrogel. Dent Mater J 2012; 31: 489-493.

19) Yasuda H, Tanabe K, Sato T, Nomoto S, Miura T, Yoshinari M. Osteogenic effect of local administration of fluvastatin using a fluvastatin-gelatin complex in senile osteoporosis model rats. J Hard Tissue Biol 2014; 23: 389-398.

20) Ohira T, Tanabe K, Sasaki H, Yoshinari M, Yajima Y. Effect of locally applied fluvastatin in low-turnover osteoporosis model mouse with femur bone defect. J Hard Tissue Biol 2015; $24: 147-154$.

21) Zhang Z, Li G, Shi B. Physicochemical properties of collagen, gelatin and collagen hydrolysate derived from bovine limed split wastes. J Soc Leath Tech Ch 2006; 90: 23-28.
22) Ogawa S, Onodera J, Honda R, Fujimoto I. Influence of systemic administration of atelocollagen on mouse livers: an ideal biomaterial for systemic drug delivery. J Toxicol Sci 2011; 36: 751-762.

23) Ishihara Y, Ueki K, Sotobori M, Marukawa K, Moroi A. Bone regeneration by statin and low-intensity pulsed ultrasound (LIPUS) in rabbit nasal bone. J Craniomaxillofac Surg 2014; 42: 185-193.

24) Hakata T, Sato H, Watanabe $Y$, Matsumoto M. Effect of formaldehyde on the physiochemical properties of gelatin capsule shells. Chem Pharm Bull (Tokyo) 1994; 42: 11381142.

25) Nakaoka R, Tabata Y, Ikada Y. Potentiality of gelatin microsphere as immunological adjuvant. Vaccine 1995; 13 : 653-661.

26) Matuda S, Se N, Iwata H, Ikada Y. Evaluation of the antiadhesion potential of UV cross-linked gelatin films in a rat abdominal model. Biomaterials 2002; 23: 2901-2908.

27) Cortesi R, Nastruzzi C, Davis SS. Sugar cross-linked gelatin for controlled release: Microspheres and disks. Biomaterials 1998; 19: 1641-1649.

28) Klawitter JJ, Hulbert SF. Application of porous ceramics for the attachment of load bearing internal orthopedic applications. J Biomed Mater Res 1971; 5: 161-229.

29) Schwartz I, Robinson BP, Hollinger JO, Szachowicz EH, Brekke J. Calvarial bone repair with porous D,L-Polylatide. Otolaryngol Head Neck Surg 1995; 112: 707-713.

30) Yamamoto M, Tabata Y, Kawasaki H, Ikada Y. Promotion of fibrovascular tissue ingrowth into porous sponges by basic fibroblast growth factor. J Mater Sci Mater Med 2000; 11: 213-218.

31) Papageorgiou GZ, Papadimitriou S, Karavas E, Georgarakis E, Docoslis A, Bikiaris D. Improvement in chemical and physical stability of fluvastatin drug through hydrogen bonding interactions with different polymer matrices. Curr Drug Deliv 2009; 6: 101-112.

32) Almeida PF, Silva LSC, Calarge FA, Brito FTM, Santana JCC. FTIR characterization of gelatin from chicken feet. J Chem Chem Eng 2012; 6: 1029-1032.

33) Ulubayram K, Eroglu I, Hasirci N. Gelatin microspheres and sponges for delivery of macromolecules. J Biomater Appl 2002; 16: 227-241.

34) Patel ZS, Yamamoto M, Ueda H, Tabata Y, Mikos AG. Biodegradable gelatin microparticles as delivery systems for the controlled release of bone morphogenetic protein-2. Acta Biomater 2008; 4: 1126-1138.

35) Pachence JM. Collagen-based devices for soft tissue repair. J Biomed Mater Res 1996; 33: 35-40. 